\title{
Texture Feature Extraction For Biometric Authentication using Partitioned Complex Planes in Transform Domain
}

\author{
Dr. Vinayak Ashok Bharadi \\ Information Technology Department \\ Thakur College of Engineering \& Technology, Mumbai University \\ Mumbai-101, Maharashtra, India
}

\begin{abstract}
Feature vector generation is an important step in biometric authentication. Biometric traits such as fingerprint, finger-knuckle prints, palmprint, and iris are rich in texture. This texture is unique and the feature vector extraction algorithm should correctly represent the texture pattern. In this paper a texture feature extraction methodology is proposed for these biometric traits. This method is based on one step transform of the two dimensional images and then using the intermediate transformation data to generate complex planes for feature vector generation. This method is implemented using Walsh, DCT, Hartley, Kekre Transform \& Kekre Wavelets. Results indicate the effectiveness of the feature vector for biometric authentication.
\end{abstract}

Keywords- Biometrics; Transforms; DCT; FFT; Kekre Transform; Hartley Transform; Kekre Wavelets.

\section{INTRODUCTION}

Biometric Authentication systems take the advantage of the uniqueness of the human body. They derive the classifying function from what a person is than what a person carries (like smartcard, token etc.). Biometrics comprises methods for uniquely recognizing humans based upon one or more intrinsic physical and/or behavioral traits. In computer science, in particular, biometrics is used as a form of identity access management and access control. It is also used to identify individuals in groups that are under surveillance [1].

Biometric characteristics can be divided in two main classes:

- Physiological are related to the shape of the body. Examples include, but are not limited to fingerprint, face recognition, DNA, Palmprint, hand geometry, iris recognition, which has largely replaced retina, and odor/scent [1], [2], [3].

- Behavioral are related to the behavior of a person. Examples include, but are not limited to typing rhythm, gait, and voice \& handwritten signatures. Some researchers have coined the term behaviometrics for this class of biometrics [2]. In this paper mainly palmprints \& iris are considered, which come under physiological biometric traits.

Palmprints and iris are rich in texture features, this information must be extracted in terms of feature vector for classification of these biometric traits. Sample of palmprint and iris from the database used for research is given below, Fig.1Shows palmprint sample from PolyU palmprint database [4] and iris image from Phoenix iris database [5].

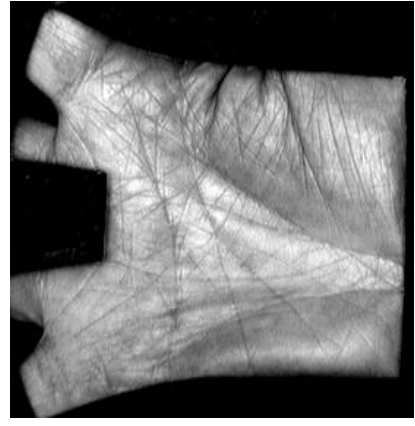

(a)

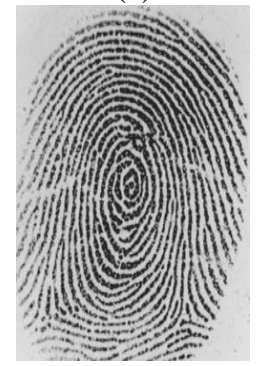

(c)

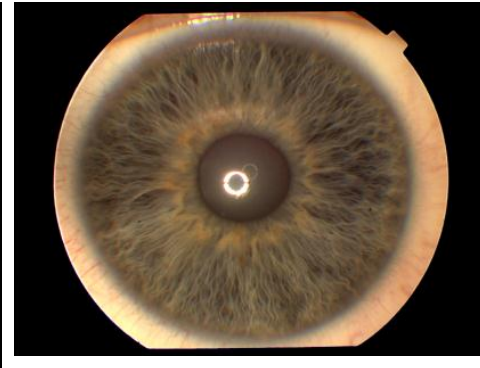

(b)

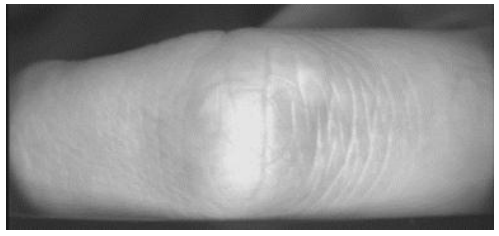

(d)
Figure 1. (a) Palmprint Image for PolyU Database [4] (b) Iris Image form Phoenix Iris Database[5]. (c) Fingerprint (d) Finger-Knuckle Print

Fingerprints, Palmprints \& iris are believed to have the critical properties of universality, uniqueness, permanence and collectability for personal authentication [1], Finger-knuckle prints are one of the emerging biometric traits [6]. What's more, palmprints have some advantages over other hand-based biometric technologies, such as fingerprints and hand geometry. Palms are large in size and contain abundant features of different levels, such as creases, palm lines, texture, ridges, delta points and minutiae. Faking a palmprint is more difficult than faking a fingerprint because the palmprint texture is more complicated; and one seldom leaves his/her complete palmprint somewhere unintentionally.

Iris also have high degree of uniqueness due to the stricture formed by muscles controlling the cornea of human eye, but higher degree of user cooperation is required in case of iris based systems [1],[6]. 
Fingerprints are used widely for authorization as well as for forensic purpose, Finger-knuckles are also rich in texture and this property can be used for biometric authentication.

In this paper we have proposed a feature vector extraction method based on intermediate Walsh transform. Where instead of taking full 2D transform, intermediate transform is taken to generate the CAL \& SAL functions of Walsh transform [7]. This information is used for generating complex Walsh plane [8] and feature vector is extracted from this. This method will be discussed in the coming section.

\section{EXISTING METHODS}

Palmprints are very rich in texture. We can form the feature vector by extracting texture information. Various approaches are followed by researchers. Pan \& Ruan [9] used 2D Gabor filters at different angles to extract the feature information. A phase based palmprint matching approach is suggested by $\mathrm{T}$. Aokit et al. [10]. They used a Band Pass phase only correlation method to extract the spectral information. Another correlation based method is presented by N. E. Othman et al. [11]. They proposed an approach based on the application of unconstrained minimum average correlation energy (UMACE) filter for palmprint feature extraction and representation [11]. The UMACE methodology determines a different filter for each palmprint of authentic class, the correlation function gives peak for authentic palmprint, and this property is used for classification.

Principal component analysis based approaches are suggested in [12], [13], [14], [15], [16]. They include PCA on PCA \& 2D PCA analysis of Gabor Wavelets, Moment invariants etc. Wavelet energy based feature vector are also possible for palmprints [17]. K. Wong, G. Sainarayanan and A. Chekima [18] used wavelet energy of the palmprint ROI. Palmprint image was decomposed using different types of wavelets for six decomposition levels. Two different wavelet energy representations were tested. The feature vectors were compared to the database using Euclidean distance or classified using feed-forward back-propagation neural network.

X. Wu, K. Wang, D. Zhang [19] used 3 level decomposition of palmprint and formed the wavelet energy based feature vector for matching. We have proposed a feature based on wavelet energy entropy. We have used Kekre's Wavelet for extraction of feature vector and the palmprint was decomposed into five levels. For classification relative wavelet energy entropy as well as Euclidian distance based classifier is used [20].

The iris texture contains information which should be extracted and represented using selected feature vector. S Attrachi \& K Faez [21] have used a complex mapping procedure and best-fitting line for the iris segmentation and 1D Gabor filter with two dimensional Principal Component Analysis (2DPCA) for the recognition approach. In the recognition procedure, they used the real term of 1D Gabor filter. In order to reduce the dimensionality of the extracted features, the new introduced 2DPCA method was used. Another such system using Gabor filter, 2DPCA \& Gabor Wavelet Neural Network (GWNN) was proposed by Zhou et al. [22].
Koh et al. have proposed multimodal iris recognition system [23] using two iris recognitions and also the levels of fusion and the integration strategies to improve overall system accuracy. This technique first implements the Daugman's iris system using the Gabor transform and Hamming distance. Second, they proposed an iris feature extraction method having a property of size invariant through the Fuzzy-LDA with five types of Contourlet transform. This gives a multimodal biometric system based on two iris recognition systems. To effectively integrate two systems, they used statistical distribution models based on matching values for genuine and impostor, respectively. Iris recognition based on linear discriminant analysis (LDA) and Linear Predictive Cepstral Coding (LPCC) was proposed by Chu \& Ching [24]. In addition, a simple and fast training algorithm, particle swarm optimization (PSO), was also introduced for training the Probabilistic Neural Network (PNN) [24].

Automatic Fingerprint Identification Systems (AFIS) try to match fingerprint by matching these ridge valley structure. Mainly two types are systems are there [1], [6], they are Minutiae based matching and Correlation based matching. Fingerprint matching has been also approached from several different strategies, like image-based [6] and ridge pattern matching of fingerprint representations. There also exist graphbased schemes [1], [3], [6], [8] for fingerprint matching. Minutiae based system try to identify the location and type of minutia and match it with database template. The accuracy is totally dependent on the identification of minutia point.

In fact, the image pattern in the finger-knuckle surface is highly unique and thus can serve as a distinctive biometric identifier [35], [36]. FKP being recent has been yet to be thoroughly explored. The current research has shown great potential in FKP to be used as an efficient and accurate biometric trait [35], [36], [37], [38]. In [38] they have proposed a local-global feature fusion for FKP verification; Local features are extracted using a bank of Gabor filters convolved with FKP ROI and global features are taken from band limited phase only correlation function. Authors have proposed use of wavelet based features [6], specifically wavelet energy of the FKP ROI for verification purpose. This is a faster approach attractive for online verification.

In this paper we are using texture based feature extraction for above mentioned biometric traits. This feature extraction mechanism is explained in the next section.

\section{PARTITIONED COMPLEX WALSh PlANE IN TRANSFORM DOMAIN}

Here we discuss a method which deals with palmprint identification in the transform domain. The one-step Walsh transform i.e. either the row or the column transforms of the fingerprint is subjected to partitioning to generate the feature vector. This process is based on Cal \& Sal Functions of Walsh Transform, next we discuss the Walsh transform \& it's Cal, Sal functions.

\section{A. Walsh Functions [25]}

Walsh functions are a set of orthogonal functions which can be used to represent any discrete-time signal. The Walsh 
functions (W0 - W7) as shown in the Fig. 2 are generated from square wave functions of different sequency.

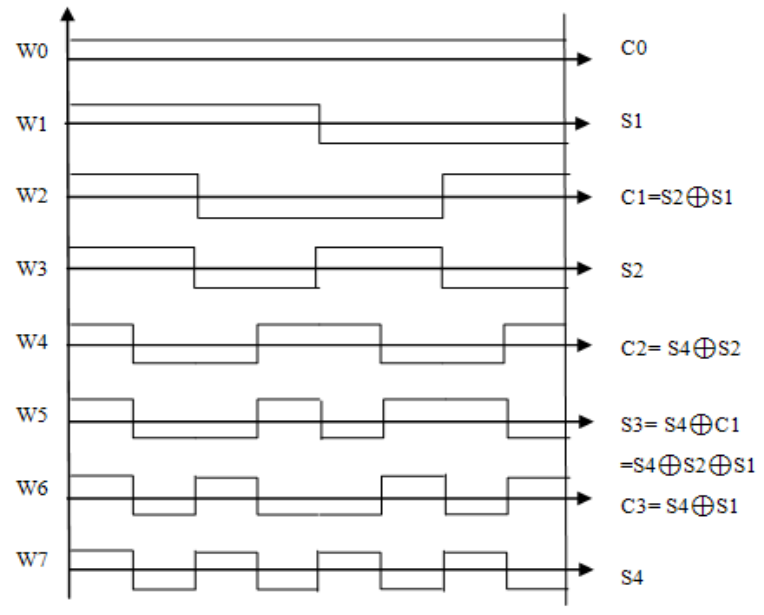

Figure 2. First Eight Walsh Functions

The even functions $(\mathrm{CO}-\mathrm{C} 3)$ are called $\mathrm{Cal}$ functions and the odd functions (S1-S4) are called Sal functions. The basic square wave functions are $\mathrm{S} 1, \mathrm{~S} 2$ and $\mathrm{S} 4$. $\mathrm{C} 0$ is DC component and the remaining functions are generated from the basic square waves by EX-OR operation (equivalent to multiplication). This operation generates only the difference sequency functions (as opposed to the case of sinusoidal signals where both difference and sum frequencies are generated) e.g. $\mathrm{C} 1=\mathrm{S} 1 \oplus \mathrm{S} 2$, here $\mathrm{S} 1$ and $\mathrm{S} 2$ being odd function, their EX-OR operation results in an even function (C1). Similarly EX-OR operation of an even and odd function generates an odd function e.g. S3 = S4 $\bigoplus \mathrm{C} 1$, which can further be simplified to $\mathrm{S} 3=\mathrm{S} 4 \oplus \mathrm{S} 2 \oplus \mathrm{S} 1$, showing that all functions are generated from the basic square waves $\mathrm{S} 1, \mathrm{~S} 2$ and S4.

Walsh functions can be ordered in a number of ways. The sequency ' $k$ ' of a Walsh function is defined as half the number of zero crossings in one cycle of the time base. Walsh functions with non-identical sequencies are orthogonal, as are the functions $\mathrm{W}(\mathrm{n}, 2 \mathrm{k})$ and $\mathrm{W}(\mathrm{n}, 2 \mathrm{k}+1)$. The product of two Walsh functions is also a Walsh function. Harmuth in [25] designates the even Walsh functions $\mathrm{Cal}(\mathrm{k})$ and the odd Walsh functions $\operatorname{Sal}(\mathrm{k})[26]$,

$$
\begin{gathered}
\text { Cal }(\mathrm{n}, \mathrm{k})=\mathrm{W}(\mathrm{n}, 2 \mathrm{k}) \\
\mathrm{Sal}(\mathrm{n}, \mathrm{k})=\mathrm{W}(\mathrm{n}, 2 \mathrm{k}+1)
\end{gathered}
$$

where ' $k$ ' is the sequency.

The Walsh transform matrix (W) is then generated by sampling these Walsh functions at the middle of the smallest time interval. The matrix, as in Eqn. (3) is obtained, which can be directly used to generate the transform coefficients of a discrete signal both of 1-D and 2-D as shown in Eqn. 1 and Eqn. 2 respectively,

$$
W=\left(\begin{array}{rrrrrrrr}
1 & 1 & 1 & 1 & 1 & 1 & 1 & 1 \\
1 & 1 & 1 & 1 & -1 & -1 & -1 & -1 \\
1 & 1 & -1 & -1 & -1 & -1 & 1 & 1 \\
1 & 1 & -1 & -1 & 1 & 1 & -1 & -1 \\
1 & -1 & -1 & 1 & 1 & -1 & -1 & 1 \\
1 & -1 & -1 & 1 & -1 & 1 & 1 & -1 \\
1 & -1 & 1 & -1 & -1 & 1 & -1 & 1 \\
1 & -1 & 1 & -1 & 1 & -1 & 1 & -1
\end{array}\right)
$$

$$
\begin{gathered}
F_{1 D}=W . f \\
F_{2 D}=W . f . W^{T}
\end{gathered}
$$

The interpretation of Walsh transform of a 2-D signal can be understood by Fig. 3, where first the row transform is calculated and then the column transform. The final output has DC component in the top left corner and the sequency components increase leftwards and downwards. In the current approach, we are first generating the intermediate transform, i.e. the row transform (or column transform) of a Region of Interest (ROI) image as shown in Fig. 4, which have DC component as its first row (or column) and higher sequency components (Sal and $\mathrm{Cal}$ ) as the following rows (or columns).

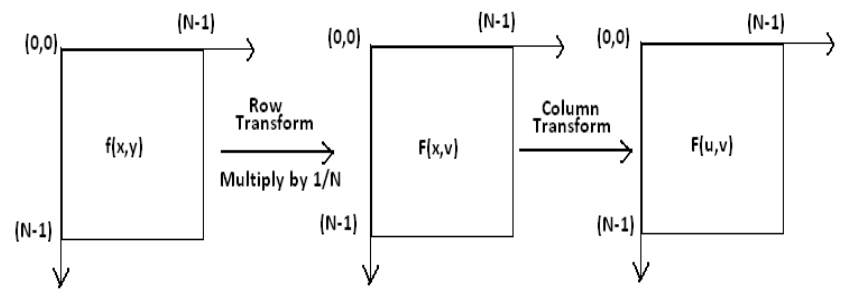

Figure 3. Transform of a 2D Function

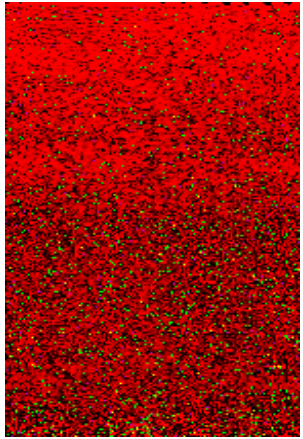

(a)

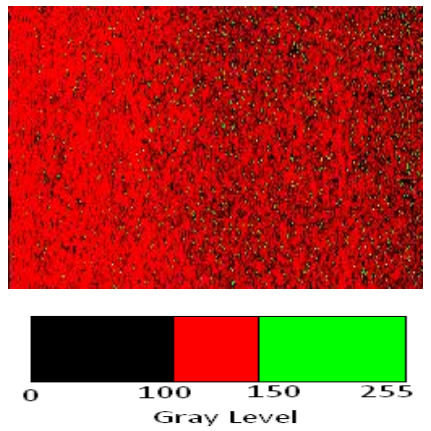

(b)
Figure 4. (a) Row Transform and (b) Column Transform of a Palmprint

\section{COMPleX Walsh Plane [27] \& Feature Vector GENERATION}

The Cal and the Sal components of the same sequency are grouped together and are considered to be in the four quadrants of 2-D complex coordinate plane as listed in Fig.5. This complex plane is now partitioned into different numbers of blocks. 
The complex plane consisting of same-sequency (Sal, Cal) components is now partitioned 256 square blocks as shown in Fig. 7. For each block a feature vector is generated which is the mean value of all the transform coefficients in that block, as well as the number of points i.e. the density is also considered.

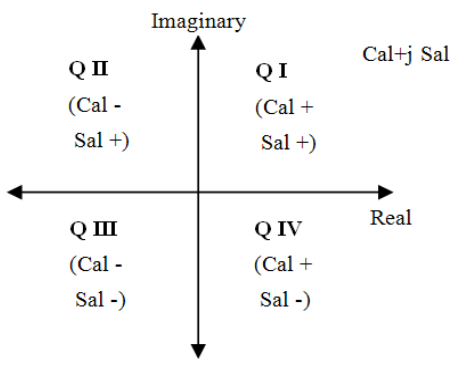

Figure 5. Complex Walsh Plane

This value is unique for each biometric trait's ROI (Region of Interest) as the sequency distribution of each ROI is unique in different blocks. As compared to all or those transform coefficients which contain major part of signal energy feature vectors generated using partitioning are much less in number and hence the reduction in processing time and complexity. The blocks generated are square shaped and the mean values of the transform coefficients in each block are calculated as in Eqn. 6, where $\mathrm{M}_{\mathrm{k}}$ is the mean and $\mathrm{N}$ is the number of coefficients in a block, which form the features. The DC component, separate means of the Sal and Cal component and the last sequency component together form the feature vector, and hence the number of features is $2 S+2$, where $S$ is the number of blocks.

$$
M_{k}=\frac{1}{N} \sum_{i=1}^{n} W_{i}
$$

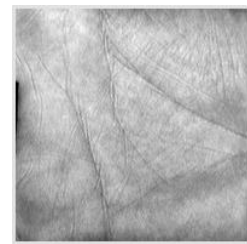

(a)

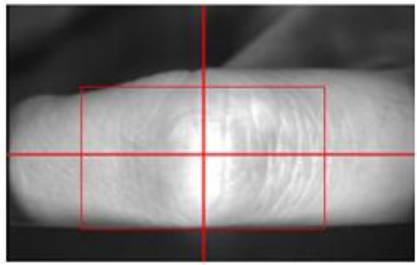

(c)

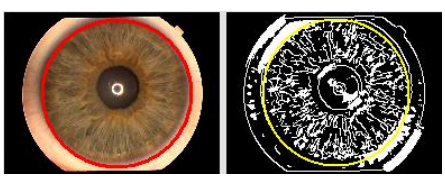

(d)

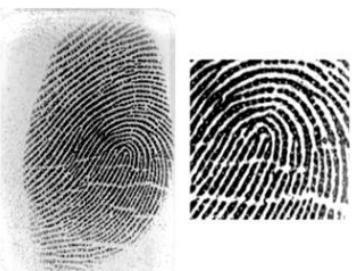

(b)
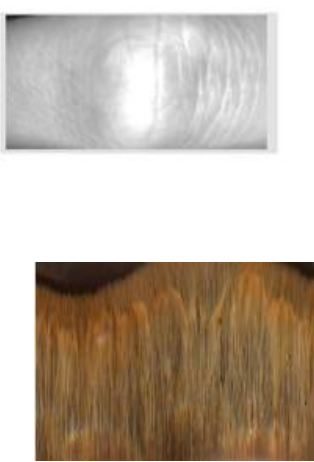

(e)
Figure 6. ROI Extraction for Palmprint \& Iris (a) Palmprint ROI (b) Fingerprint ROI(c) Finger-knuckle Print ROI (d) Iris Localization (e) Unwrapped Iris ROI
The features obtained from the test image are compared with those obtained from the stored Biometric trait in the database and the results matched. The Euclidian distances between the feature vectors of the test image and the database images are calculated. The minimum distance gives the best match.

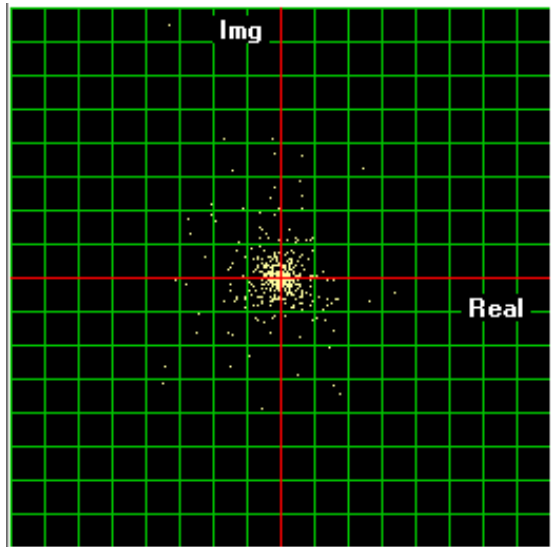

(a)

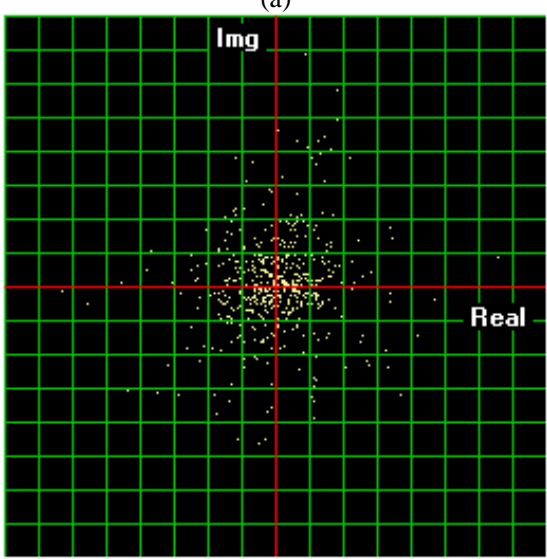

(b)

Figure 7. Complex Walsh Plane (a) Partitioned Cal+jSal Function Plot of Row

Transform (b) Partitioned Cal+jSal Function Plot for Column Transform

We have selected $192 * 192$ pixels size region of interest for the palmprint as discussed by Kekre \& Bharadi [31]. The Fingerprint ROI is $144 * 144$ pixels in size, The Finger-knuckle print ROI is $256^{*} 128$ pixels size. The iris image is also localized and ROI of size $240 * 360$ pixels is selected by unwrapping of the localized iris [28]. These ROI's are shown in Fig. 6. All of these ROI's are used for intermediate transform \& complex plane generation. While taking the intermediate transform scaling at appropriate level is done wherever required.

As discussed earlier each plot gives $2 \mathrm{~S}+2$ coefficients, we have 256 blocks in each plot, hence one plot gives $514(256 * 2$ +2 ) coefficients. For each type of input i.e. segmented ROI we have two plots, one for row and one for column transform hence we have $1028(514 * 2)$ coefficients for each type of fingerprint input. Finally we have 1028 coefficients in the feature vector of ROI. Similar Feature vector is generated for Density of the points in complex Walsh Plane for each fingerprint input. This feature vectors are used for enrollment and matching of the biometric traits displayed in Fig. 6. For 
each normalized iris ROI input the feature vector is generated in following variations, for Iris Left (L) \& Right (R) iris ROI's are considered. For Palmprint, Finger-knuckle prints \& fingerprints only first five feature vector variations are considered.

1. Row transform feature vector (Row TRF -L, Row TRF -R)

2. Column transform feature vector (Col TRF -L, Col TRF -R)

3. Row density feature vector (Row-Density-L, Row-Density-R)

4. Column density feature vector (Col-Density-L, Col-Density-R)

5. Fusion of above mention feature vectors with DC \& Sequency components.

(Row TRF + Density + DC SEQ Left, Row TRF + Density + DC SEQ Right)

6. Final Fusion of Left \& Right Iris Feature Vectors(Fusion)

The extracted feature vectors are stired in database. We are using K-NN classifier classification. We are using Hongkong Polytechnic University's POLYU Database [29] for palmprint $\&$ Finger-knuckle print testing. Phoenix iris database [30] is used for iris testing. The results are discussed in the next section.

\section{RESUlts}

We have enrolled total 100 persons in the database, 6 palmprints per person are used for training. Total 358 tests are performed for intra class matching and 2491 tests are performed for inter class matching. Similar method is followed for other biometrics also. For iris we use left as well as right iris sample for enrollment. We are using Performance Index (PI) as a metric for performance comparison the crossover rate (EER) for FAR-FRR (False Acceptance Rate \& False Rejection Rate) analysis is found and PI is defined as:

$$
\mathrm{PI}=100-\mathrm{EER}
$$

Higher the PI, better is the performance of specific feature vector extraction mechanism. The details of PI \& CCR (Correct Classification Ratio) of above mentioned feature vectors are summarized in Fig. 8, $9 \&$ 10. Fig. 8 shows FARFRR Analysis of fusion based feature vector. We have achieved $10 \%$ EER.

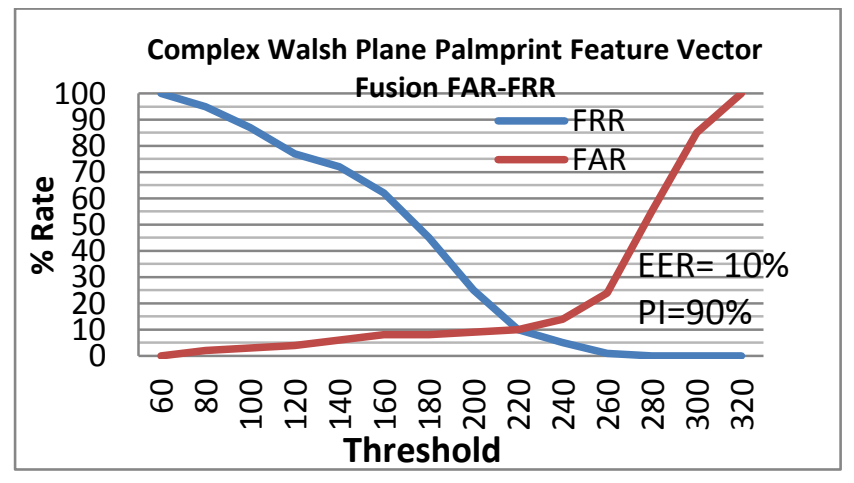

Figure 8. FAR-FRR Analysis for Walsh Cal-Sal based Fused Feature Vector
Fusion of Row \& Column Transform mean \& Density with DC \& Sequency coefficient gives 90\% PI. The individual Row $\&$ column transform mean based feature vectors have $82 \%$ \& $87 \%$ PI, this shows that due to fusion of feature vector with DC \& Sequency component the performance has improved.

Fig. 9 shows comparative analysis of the different variations of the feature vectors. The correct classification ratio (CCR) for the matching tests is $84.23 \%$ In case of palmprint the $\mathrm{CCR}$ is lower than fingerprint; this is due to the fact that the database images have prominent details about major principle lines and the ridge and minute lines on the palms are not captured properly in the database image. The fingerprints are scanned at higher resolution of 500dpi and the PolyU palmprint database images are captured by a CCD camera at $75 \mathrm{dpi}$. In the next section a new biometric called as finger-knuckle print is discussed.

Fig. 10 shows the analysis of results for fingerprint recognition using above mentioned method extended for Hartley transform, DCT, Kekre Transform \& Kekre Wavelets. The recognition is performed with full fingerprint as well as fingerprint ROI (Core Point based). The fusion of feature vector gives best PI. Maximum performance is given by Walsh transform followed by Hartley \& Kekre Wavelet Transform.

Fig. 11 shows Performance Comparison of Partitioned Complex Walsh Plane Based Fingerprint, Palmprint \& FKP Matching Techniques, It is shows that the multi-algorithmic fusion based feature vector gives best performance (Last Columns) and among the three hands based biometric traits, fingerprints give the best performance.

Fig. 12 \& 13 show the results for Iris recognition. For this biometric we have performed study in two modes. Multialgorithmic feature vector consisting of the fusion of partitioned complex plane based feature vectors, and multiinstance feature vector consisting of left \& right iris. Multiinstance feature vector gives best performance; Walsh transform based feature vector gives highest accuracy followed by DCT \& Kekre Wavelets.

\section{CONCLUSION}

In this paper we have discussed a feature extraction mechanism based on partitioned complex plane of Walsh transform along with its extension to Hartley, DCT, Kekre Transform \& Kekre Wavelets. This feature extraction mechanism was implemented for Fingerprints, Finger-knuckle prints, palmprint $\&$ iris feature vector generation.

It is observed that the proposed method performs well for classification of genuine and forgery inputs.

Best performance was given by Walsh transform followed by Kekre wavelet DCT etc.This shows the superiority of proposed method for texture feature based feature vector extraction.

The proposed method is tested in both unimodal \& multialgorithmic (involving fusion of multiple feature vectors ) \& multi-instance (involving left \& right iris sample instances). Fusion improves the performance the various biometric systems tested. 


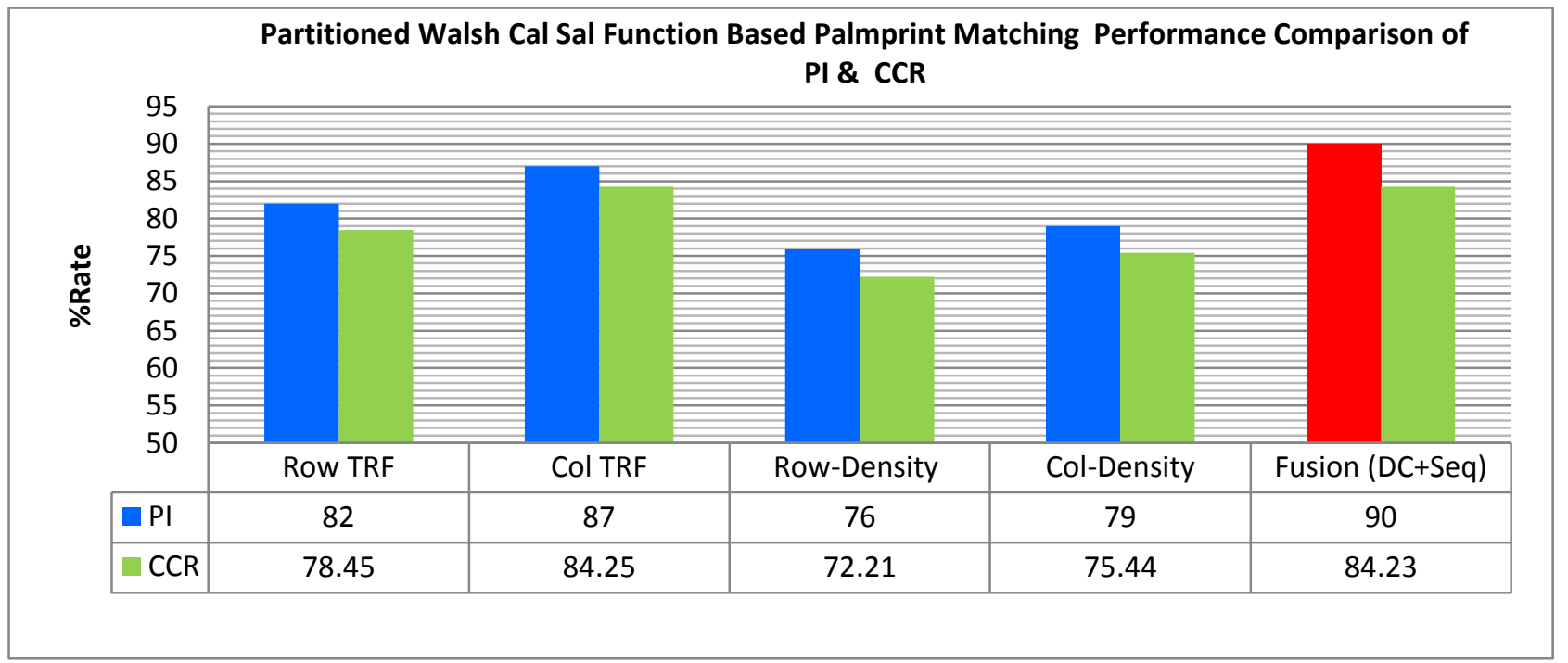

Figure 9. Performance Comparison for Feature Vector Variants of Partitioned Walsh Cal-Sal Function Palmprint Matching

Score Fusion based Matching Gives Higher Performance Index; this is Indicated by Bar in Red Colour. (TRF: Transform, DC-DC Coefficient, Seq- Sequency)

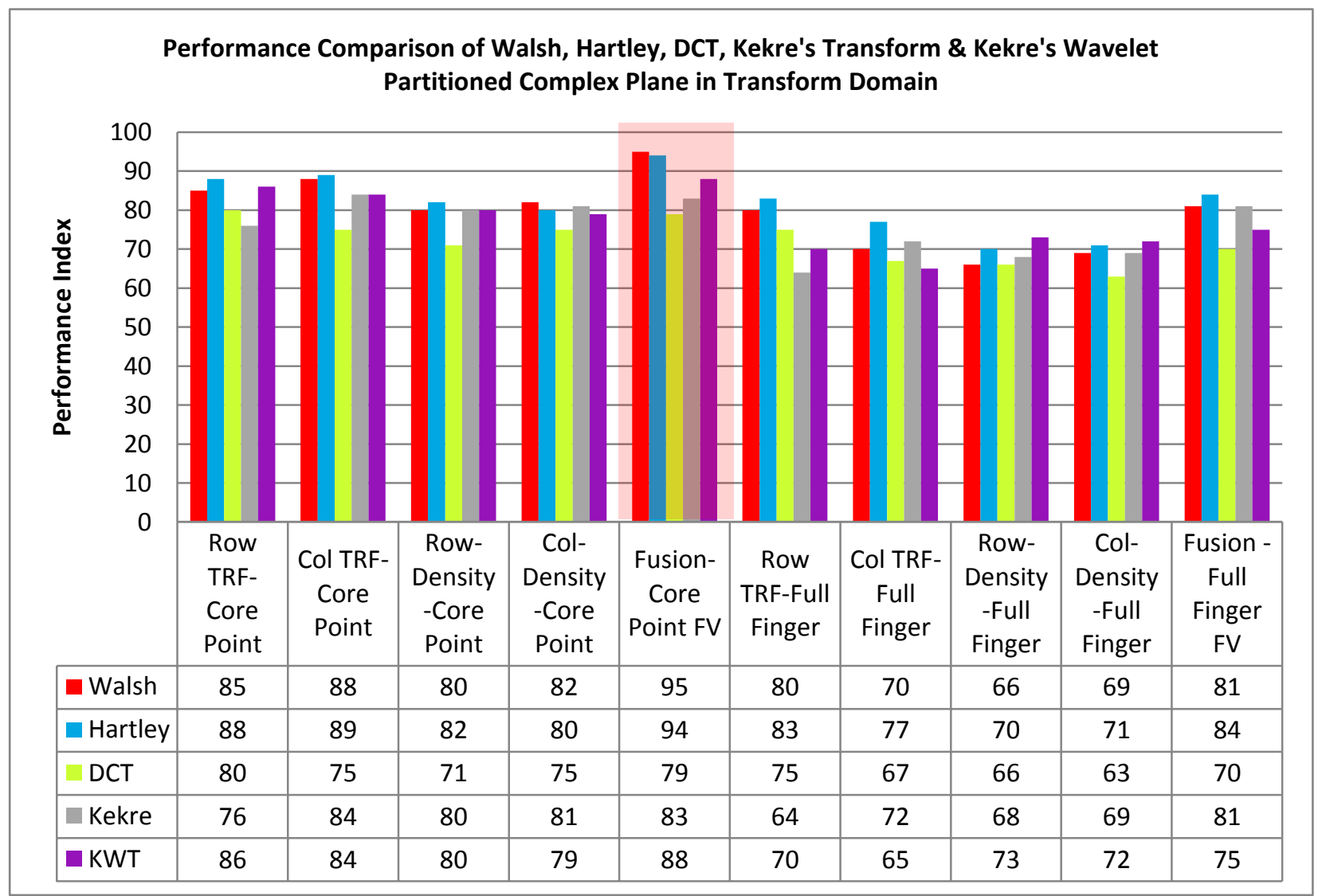

Figure 10. Performance Comparison for Feature Vector Variants of Partitioned Walsh, Hartley, DCT, Kekre's Transform \& Kekre's Wavelet. (Performance Index is Compared) 


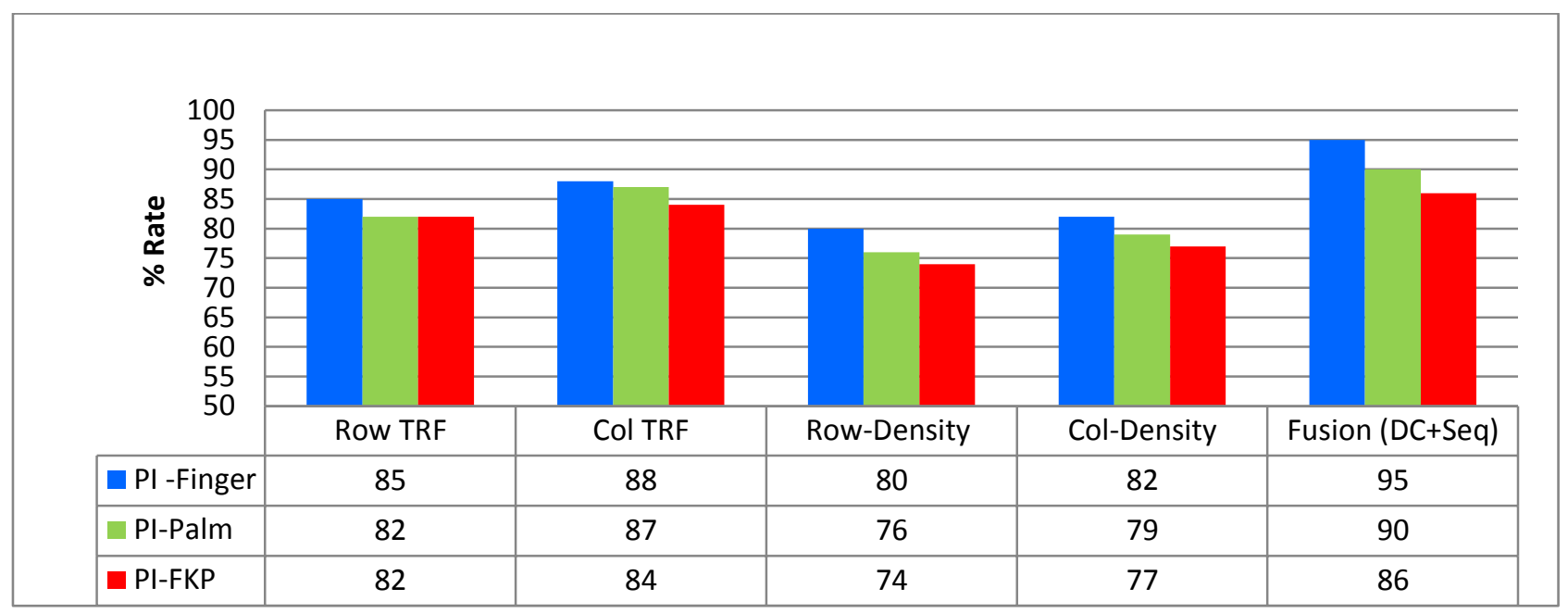

Figure 11. Performance Comparison of Partitioned Complex Walsh Plane Based Fingerprint, Palmprint \& FKP Matching Techniques

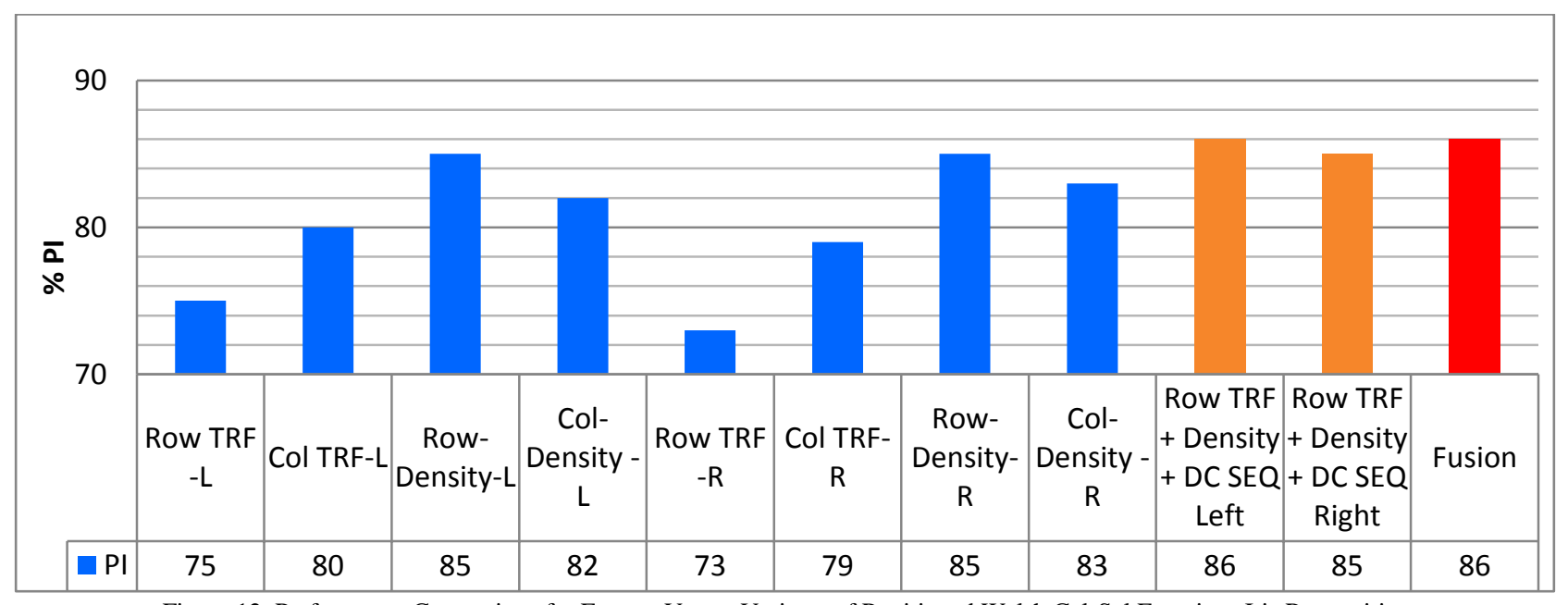

Figure 12. Performance Comparison for Feature Vector Variants of Partitioned Walsh Cal-Sal Functions Iris Recognition

Score Fusion based Matching Gives Higher Performance this is Indicated by Bar in Red Colour. The Orange Colour Bar Indicate the PI for Individual Left and Right Iris Feature Vector Fusion (TRF: Transform, FV: Feature Vector)

\begin{tabular}{|c|c|c|c|c|c|}
\hline \multirow{6}{*}{ 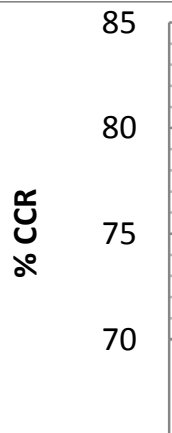 } & & & & 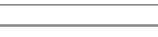 & 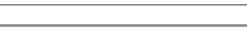 \\
\hline & & & & 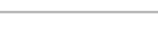 & 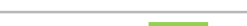 \\
\hline & & & & & \\
\hline & & & & & \\
\hline & & & & & \\
\hline & Walsh Transform & $\begin{array}{l}\text { Hartley } \\
\text { Transform }\end{array}$ & DCT & $\begin{array}{c}\text { Kekre's } \\
\text { Transform }\end{array}$ & Kekre's Wavelet \\
\hline Left Iris & 82 & 79.27 & 78.27 & 77.2 & 80 \\
\hline$\square$ Right Iris & 82.79 & 78.1 & 78.95 & 78.01 & 77.98 \\
\hline Fusion & 84 & 74.4 & 81.52 & 74 & 80.53 \\
\hline
\end{tabular}

Figure 13. Performance Comparison for Feature Vector Variants of Partitioned Complex Plane based Iris Feature Vectors 


\section{ACKNOWLEDGMENT}

Sincere thanks to L. Zhang, L. Zhang, and D. Zhang from Hongkong Polytechnic University for allowing the use of biometric database.

\section{REFERENCES}

[1] A. K. Jain, A. Ross, S. Prabhakar, "An Introduction to Biometric Recognition", IEEE Transactions on Circuits and Systems for Video Technology, Vol. 14, No. 1, January 2004

[2] H. B. Kekre, T. K. Sarode, R. Vig, "Fingerprint Matching using Sectorized Complex Walsh Plane in Transform Domain", Proceedings of ICWET 2011, ACM Conference, Mumbai, India, Feb. 2011

[3] H. B. Kekre, V. A. Bharadi , "Using Component Object Model for Interfacing Biometrics Sensors to Capture Multidimensional Features", International Journal of Intelligent Information Technology Application JIITA), ISSN 1999-2459, Vol.2 , No. 6, pp. 279-285, December 2009

[4] Hong Kong University PolyU Database : www4.comp.polyu. edu.hk/ biometrics/2D_3D_Palmprint.htm

[5] Phoenix Iris Database: http://phoenix.inf.upol.cz/iris/ download

[6] H. B Kekre, V A Bharadi, "Biometric Authentication Systems", Ph. D. Thesis Submitted to NMIMS University, June 2011

[7] H. F. Harmuth, "Applications of Walsh Functions in Communications." IEEE Spectrum 6, pp. 82-91, 1969

[8] H. B. Kekre, T. K. Sarode, R. Vig, "Fingerprint Matching using Sectorized Complex Walsh Plane in Transform Domain", Proceedings of ICWET 2011, ACM Int. Conference, Mumbai, India, Feb. 2011

[9] X. Pan, Q. Ruan, "A Modified Preprocessing Method for Palmprint Recognition", In Proceedings of 8th International Conference on Signal Processing, 2006, IEEE DOI : 0-7803-9737-1/06

[10] K. Ito, T. Aokit, H. Nakajima, K. Kobayashi,T. Higuchi, "A Palmprint Recognition Algorithm Using Phase-Based Image Matching", In proceedings of IEEE Int. Conference on IP, pp. 2669 - 2672, 2006

[11] N. E. Othman, A. A. Azid, S. Samad and A. Hussain, "A Palmprint Recognition System using Correlation Filters", In Proceedings of 4th Student Conference on R\&D (SCOReD 2006), Selangor, Malaysia,pp.91-94, June 2006

[12] M. Laadjel, A. Bouridane, F. Kurugollu, "Eigenspectra Palmprint Recognition", Proceedings of 4th IEEE International Symposium on Electronic Design, Test \& Applications, pp.382-385, 2008

[13] M. Ekinci and M. Aykut, "Gabor-based Kernel PCA for Palmprint Recognition", Electronics Letters, Vol. 43 No. 20, 27th September 2007

[14] M. You, S. Jifeng, "Palmprint Recognition Based on 2DPCA-Moment Invariant", In Proceedings of 5th International Conference on Image and Graphics 2009, IEEE: DOI 10.1109/ICIG.2009.168, pp.149-155,2009

[15] C. Wen, J. Zhang, "Palmprint Recognition based on Gabor Wavelets and 2-Dimensional PCA \& PCA", In Proceedings of the 2007 International Conference on Wavelet Analysis and Pattern Recognition, Beijing, China, pp. 1518 - 1523, Nov. 2007

[16] S. Iitsuka, K. Miyazawa, T. Aoki, "A Palmprint Recognition Algorithm Using PCA Of Phase Information",ICIP'09 Proceedings of the 16th IEEE Int. Conf. on Image, ICIP 2009, pp.1973-1976,2009

[17] S. Iitsuka, K. Miyazawa,T. Aoki, "Palmprint Identification Using Wavelet Energy", International Conference on Intelligent and Advanced Systems 2007, pp. 714 - 719, 2007

[18] K. Wong, G. Sainarayanan, A. Chekima, "Palmprint Identification Using Wavelet Energy", In Proceedings of International Conference on Intelligent and Advanced Systems 2007, pp.714-719,2007
[19] X. Wu, K. Wang, D. Zhang, "Wavelet Based Palmprint Recognition", Proceedings of the First International Conference on Machine Learning and Cybernetics, Beijing, 4-5 November 2002, pp. 1256-1257,2002

[20] H B Kekre, V A Bharadi, "Palmprint Recognition Using Kekre's Wavelet's Energy Entropy Based Feature Vector", Proceedings of ICWET 2011, TCET, Mumbai, India, pp. 39-45, Feb. 2011

[21] S. Attarchi, K. Faez, A. Asghari, "A Fast and Accurate Iris Recognition Method Using the Complex Inversion Map and 2DPCA", 7th IEEE/ACIS International Conference on Computer and Information Science, ICIS 08, pp. 179 - 184,2008

[22] Z. Zhou, H. Wu , Q. Lv,"A New Iris Recognition Method Based on Gabor Wavelet Neural Network", International Conference on Intelligent Info. Hiding and Multimedia Sig. Proc, pp. 1101 - 1104, Aug. 2008

[23] H. Koh, W. Lee, M. Chun, "A Multimodal Iris Recognition Using Gabor Transform and contourlet",2nd International Conference on Signal Processing and Comm. Systems, ICSPCS 2008, pp. 1 - 6, Dec. 2008

[24] C. Ching, C. Chen," High Performance Iris Recognition Based on LDA and LPCC",17th IEEE International Conference on Tools with Artificial Intelligence (ICTAI’05),pp. pp. 421-426, 2005

[25] H. F. Harmuth, "Applications of Walsh Functions in Communications." IEEE Spectrum 6, pp. 82-91, 1969

[26] E. W. Weisstein, "Walsh Function", From MathWorld-A Wolfram Web Resource, http://mathworld.wolfram.com/ WalshFunction.html

[27] H. B. Kekre, T. K. Sarode, R. Vig, "Fingerprint Matching using Sectorized Complex Walsh Plane in Transform Domain", Proceedings of ICWET 2011, ACM Int. Conference, Mumbai, India, Feb. 2011

[28] L. Ma, T. Tan, Y. Wang, and D. Zhang, "Efficient Iris Recognition by characterizing Key Local Variations", IEEE Transaction on IP, Vol. 13, No.6, pp. 739-750, 2004

[29] Hong Kong University PolyU Database : www4.comp.polyu. edu.hk/ biometrics/2D_3D_Palmprint.htm

[30] Phoenix Iris Database: http://phoenix.inf.upol.cz/iris/ download/

[31] H. B. Kekre, V. A. Bharadi , "Palmprint Recognition Using Kekre's Wavelet's Energy Entropy Based Feature Vector", ACM International Conf., ICWET 2011, India, pp.39-45, Feb. 2011

[32] H. B. Kekre, A. Athawale, D. Sadavarti, "Algorithm To Generate Kekre's Wavelet Transform from Kekre's Transform", IJSET, June 2010

[33] H. B. Kekre, V. A. Bharadi , "Performance Comparison of DCT, FFT, WHT, Kekre's Transform \& Gabor Filter Based Feature Vectors for On-Line Signature Recognition", Int. Journal of Computer Application (IJCA), Special Issue for ACM Conf. ICWET 2011, February 2011

[34] H. B. Kekre, V. A. Bharadi, "Hybrid Multimodal Biometric Recognition Using Kekre's Wavelets, 1D Transforms \& Kekre's VQ Algorithms Based Feature Extraction of Face \& Iris", IJCA, Special Issue for ACM International Conference ICWET 2011, February 2011

[35] http://www.comp.polyu.edu.hk/ biometrics/FKP.htm

[36] L. Zhang, L. Zhang, and D. Zhang, "Finger-Knuckle Print Verification Based On Band-Limited Phase-Only Correlation”, CAIP'09, 2009

[37] H. B. Kekre, V. A. Bharadi, "Finger-Knuckle-Print Region of Interest Segmentation using Gradient Field Orientation \& Coherence", Proceedings of IEEE Int. Conf. ICETET 2010, pp. 43-50, Dec. 2010

[38] Lin Z., Lei Z., David Z., H. Zhub, "Ensemble of Local and Global Features for Finger-Knuckle-Print Recognition", Biometrics Research Center, Department of Computing, The Hong Kong Polytech. University

\section{Authors PROFILE}

Dr. Vinayak Ashok Bharadi has received B.E. Electronics Engg.in 2002 \& M. E. Electronics \& Telecomm in 2007 from Mumbai University. He has completed Ph.D. in Engineering (Biometrics Authentication Systems) from NMIMS University in 2011. He has worked Over 9 years as a faculty of Electronics \& I T Engg. He has published 50+ National \& International papers. 